\title{
Three-nucleon force effects in proton-deuteron scattering
}

\author{
S. Ishikawa ${ }^{1, a}$ \\ Science Research Center, Hosei University, 2-17-1 Fujimi, Chiyoda, Tokyo 102-8160, Japan
}

\begin{abstract}
Possible three-nucleon forces that may be considered in addition to a widely accepted two-pion exchange three-nucleon force are studied to improve the fit to experimental data of proton-deuteron scattering. Calculations are performed with including effects of long-range Coulomb force by a recently developed method to solve the Faddeev equation in coordinate space. Two different kinds of three-nucleon potentials: a phenomenological potential and one due to the exchange of pion and sigma bosons, are presented and compared with experimental data of elastic differential cross sections, a tensor analyzing power, and a nucleon-to-nucleon polarization transfer coefficient.
\end{abstract}

\section{Introduction}

In a conventional picture that atomic nuclei are quantum mechanical systems consisting of nucleons, interaction potentials among the nucleons are basic ingredients to construct the nuclear Hamiltonian. In the middle of 1990's, a number of realistic nucleon-nucleon potential (2NP) models that describe nucleon-nucleon (NN) experimental data with a high accuracy $\left(\chi^{2} /\right.$ datum $\left.\sim 1\right)$ were proposed. Examinations of these models for observables of few-nucleon systems with accurate calculations showed that there exist some discrepancies between calculations and experimental data. For example, calculations of the binding energies of three-nucleon $(3 N)$ bound states, ${ }^{3} \mathrm{H}$ and ${ }^{3} \mathrm{He}$, are smaller compared to empirical values, and calculated differential cross section minima of nucleon-deuteron $(N d)$ elastic scattering are too small compared to experimental data. A possible improvement of the nuclear Hamiltonian to get rid of these discrepancies is to introduce threenucleon forces (3NFs). A $3 \mathrm{NF}$ that is originated from the exchange of two pions among three nucleons, the two-pion exchange three-nucleon force $(\pi \pi-3 \mathrm{NF})$, was considered as a long-range component of $3 \mathrm{NFs}$, and was found to successfully solve the problems of the binding energy and the differential cross sections. However, recent studies show that discrepancies in some polarization observables, such as the vector analyzing power $A_{y}(\theta)$ and the tensor analyzing power $T_{21}(\theta)$ in the elastic $N d$ scattering, are not explained by the introduction of the $\pi \pi-3 N F$. (See Fig. 1 below for an example.) This demonstrates our little understanding on spin-dependence of $3 \mathrm{NFs}$ and a need to consider additional mechanisms to introduce different spindependent $3 \mathrm{NFs}$ from the $\pi \pi-3 \mathrm{NF}$.

In this paper, we present a possible improvement of the nuclear Hamiltonian by considering two different 3NFs: a purely phenomenological one and a theoretical one based on the boson-exchange picture of the nuclear interaction.

\footnotetext{
a e-mail: ishikawa@hosei.ac.jp
}

After giving some technical notes about our $3 N$ calculations including long-range effects of the proton-proton $(p p)$ Coulomb interaction in Sec. 2, a phenomenological 3NF that is made to reproduce low-energy $3 N$ observables, is introduced in Sec. 3, In Sec. 4, as a possible mechanism to produce a $3 \mathrm{NF}$ similar to the phenomenological $3 \mathrm{NF}$ in the preceding section, the exchange of a pion and a scalarisoscalar boson $(\sigma)$ among three nucleons is introduced and its effects on $3 N$ scattering observables are discussed. A summary is given in Sec. 5 .

\section{Three-nucleon calculations including Coulomb force effects}

It is currently the most practical way of extracting useful information on $3 \mathrm{NFs}$, especially on their spin-dependence, to compare rigorous calculations of $3 \mathrm{~N}$ observables using various $3 \mathrm{NF}$ models with precision experimental data. Proton-deuteron $(p d)$ scattering experiments have a technical advantage over neutron-deuteron ones because of the availability of precise measurements with charged particles. On the other hand, due to a mathematical difficulty in treating three-body systems with the long-range Coulomb interaction, calculations of the $p d$ scattering at energies above the three-body breakup threshold are one of the most challenging subjects in physics of few-body systems.

In the last decade, there have been some developments in this problem by calculations based on the Kohn variational principle [1] and on momentum space Faddeev equations using the screening and renormalization method [2]. Calculations performed in this paper are based on different approach from these: to solve Coulomb-modified Faddeev equations [3] as an integral equation form in coordinate space, which was successfully applied for the $3 N$ bound state $\left({ }^{3} \mathrm{He}\right)$ [4] and the $p d$ scattering problem below [5] and above [6,7] the breakup threshold. In this approach, an auxiliary potential Coulomb potential acting between a 
proton and the center of mass of the rest proton-neutron pair, is introduced to cancel out the long-rangeness of the $p p$ Coulomb potential in the integral kernel. Although the cancellation is not perfect due to the appearance of threebody breakup components of the reaction, it turns out that a satisfactory result is obtained by taking into account only short-range parts of the Coulomb contribution by multiplying with a damping factor in the integral kernel. Some details of this approach as well as its accuracy and convergence are described in Ref. [7].

In our calculations below, partial wave states with the total angular momentum of two-body sub-system up to 6 for bound states and up to 4 for scattering states, and partial wave states with the $3 \mathrm{~N}$ total angular momentum up to $19 / 2$ are taken into account.

We use the Argonne $\mathrm{V}_{18}$ model (AV18) [8] as a standard 2NF, which gives $6.928 \mathrm{MeV}(7.626 \mathrm{MeV})$ for the ${ }^{3} \mathrm{He}\left({ }^{3} \mathrm{H}\right)$ binding energy in contrast with the empirical value of $7.718 \mathrm{MeV}(8.482 \mathrm{MeV})$. We use a new version of the Brazil model (BR) [9] for the $\pi \pi-3 N F$. In the Brazil model, a cutoff mass $\Lambda_{\pi}$ in the dipole $\pi N N$ form factor, (see Eq. (6) below,) is a parameter to be determined. When one wants to reproduce the empirical value of $3 \mathrm{~N}$ binding energy in the combination of the AV18-2NF and the BR-3NF, one needs to choose the cutoff mass $\Lambda_{\pi}$ to be 660 $\mathrm{MeV}\left(\mathrm{AV} 18+\mathrm{BR}_{660}\right)$. This value is rather small compared to ones often used in one-boson exchange models for $2 \mathrm{NP}$ models, which are more than $1 \mathrm{GeV}$. Since calculations of the $3 \mathrm{~N}$ binding energy strongly depend on the value of $\Lambda_{\pi}$ used, the use of larger values for $\Lambda_{\pi}$ produces an overbinding problem of the $3 N$ system. For example, if we choose a value of $800 \mathrm{MeV}$ for $\Lambda_{\pi}\left(\mathrm{AV} 18+\mathrm{BR}_{800}\right)$, we get 8.913 $\mathrm{MeV}$ for the ${ }^{3} \mathrm{He}$ binding energy, which is larger than the empirical value by about $1 \mathrm{MeV}$. This implies the existence of additional $3 \mathrm{NFs}$, which may produce repulsive effects in the $3 \mathrm{~N}$ bound states.

As an example of polarization observables, calculations of the tensor analyzing power $T_{21}(\theta)$ are compared with experimental data [10] in Fig. 1. The effect of the $\pi \pi-3 \mathrm{NF}$, which is designated by the difference between the solid curve (AV18) and the dashed curve ( $\left.\mathrm{AV} 18+\mathrm{BR}_{660}\right)$, is visible for a range of the scattering angles up to about $150^{\circ}$. While the $\pi \pi-3 \mathrm{NF}$ works to improve a fit to the experimental data of the $T_{21}(\theta)$ around $\theta=120^{\circ}$, it shifts the calculation away from the experimental data around $\theta=90^{\circ}$. In Ref. [11], it is shown that this undesirable contribution of the $\pi \pi-3 \mathrm{NF}$ to $T_{21}(\theta)$ occurs even at low energies below the breakup threshold, and is due to a spin tensor component in the $\pi \pi-3 \mathrm{NF}$.

\section{Phenomenological 3NF}

The successful and unsuccessful results of the $\pi \pi-3 N F$ to reproduce $3 N$ observables indicate that we need to consider another kinds of $3 \mathrm{NFs}$ in addition to the $\pi \pi-3 \mathrm{NF}$. It may be convenient to understand what kind of spin dependence should be required to reproduce unsuccessful (polarization) observables. As a first step to study relations between spin characteristics of $3 \mathrm{NFs}$ and $3 N$ observables,

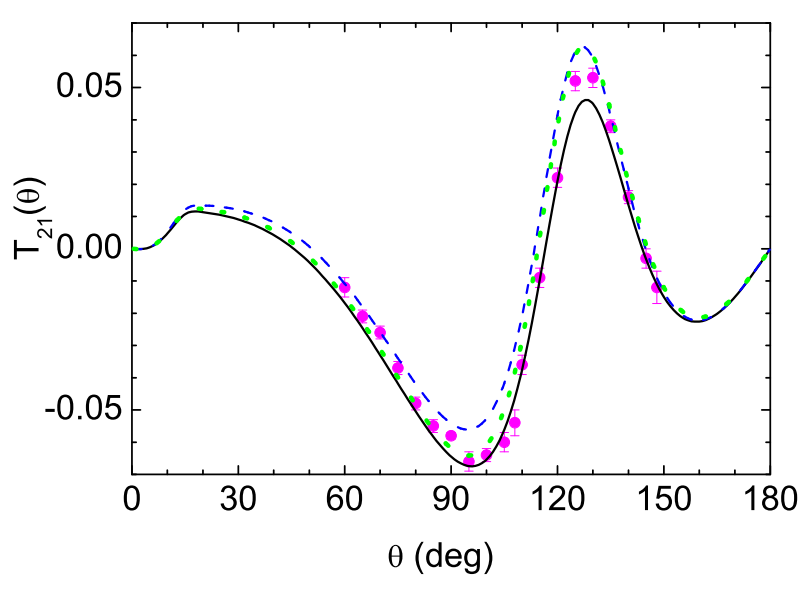

Fig. 1. Tensor analyzing power $T_{21}(\theta)$ of the $p d$ elastic scattering at $E_{p}=10.0 \mathrm{MeV}$ (or equivalently $E_{d}=20.0 \mathrm{MeV}$ ). The solid (black) curve denotes the calculation with AV18, the dashed (blue) curve that with $\mathrm{AV} 18+\mathrm{BR}_{660}$, and the dotted (green) curve that with $\mathrm{AV} 18+\mathrm{BR}_{800}+V^{\text {phe }}$. Experimental data are taken from Ref. [10].

we have introduced a phenomenological 3NP model [12], which has a form that typical components in 2NF: central, tensor, and spin-orbit components, are modified in the presence of third nucleon. The explicit form of the $3 \mathrm{NP}$ is

$$
\begin{aligned}
V^{p h e}= & \sum_{i<j} e^{-\left(\frac{r_{i k}}{r_{G}}\right)^{2}-\left(\frac{r_{j k}}{r_{G}}\right)^{2}}\left[V_{0}+V_{T} S_{T}(i j) \hat{P}_{11}\right] \\
& +V_{l s} e^{-\alpha_{l s} \rho} \sum_{i<j}\left[\ell_{i j} \cdot\left(\mathbf{S}_{i}+\mathbf{S}_{j}\right)\right] \hat{P}_{11},
\end{aligned}
$$

where $S_{T}(i j)$ is the tensor operator acting between the nucleon pair $i j, \hat{P}_{11}$ is the projection operator to the spin and isospin triplet state of the pair $i j$, and $\rho^{2}=$ $\frac{2}{3}\left(r_{12}^{2}+r_{23}^{2}+r_{31}^{2}\right)$ with $\mathbf{r}_{i j}=\mathbf{r}_{j}-\mathbf{r}_{i}$. We take the range parameters $r_{G}=1.0 \mathrm{fm}$ and $\alpha_{l s}^{-1}=1 / 1.5 \mathrm{fm}$.

The strength parameters, $V_{0}, V_{T}$, and $V_{l s}$, are determined to reproduce the following observables in conjunction with the AV18 $+\mathrm{BR}_{800}$ potential: the ${ }^{3} \mathrm{He}$ binding energy, the vector analyzing power $A_{y}(\theta)$ and the tensor analyzing power $T_{21}(\theta)$ of the $p d$ scattering at $E_{p}=3.0$ $\mathrm{MeV}$. It is noted that $T_{21}(\theta)$ and $A_{y}(\theta)$ depend almost individually on $V_{T}$ and $V_{l s}$, respectively, at such a low energy. Thus, after determining the parameters $V_{T}$ and $V_{l s}$ from $T_{21}(\theta)$ and $A_{y}(\theta)$, respectively, we are able to determine the parameter $V_{0}$ to reproduce the ${ }^{3} \mathrm{He}$ binding energy. A phenomenological $3 \mathrm{NF}$ with resulting parameters: $V_{0}=36$ $\mathrm{MeV}, V_{T}=-40 \mathrm{MeV}$, and $V_{l s}=-16 \mathrm{MeV}$, will be denoted as $V^{\text {phe }}$

A result of $T_{21}(\theta)$ at $E_{p}=10.0 \mathrm{MeV}$ with above set of potentials $\left(\mathrm{AV} 18+\mathrm{BR}_{800}+V^{\text {phe }}\right)$ is shown by the dotted curve in Fig. 1. The figure shows that the $V^{\text {phe }}$ is still successful in reproducing the tensor analyzing power $T_{21}(\theta)$ at a higher energy, although there is no a priori reason for that. This success demonstrates that an improvement of a tensor component in $3 \mathrm{NF}$ is a key to reproduce the tensor analyzing power $T_{21}(\theta)$ at higher energies. 
However, a limitation due to the phenomenological character of $V^{\text {phe }}$ is also observed. As an example for such cases, calculations of a nucleon-to-nucleon polarization transfer coefficient $K_{y}^{y^{\prime}}(\theta)$ in ${ }^{2} \mathrm{H}(\vec{p}, \vec{p})^{2} \mathrm{H}$ reaction as well as experimental data [13] are shown in Fig. 2. In the figure, while a deviation from the experimental data of the calculation with the AV18-2NF only (the solid curve) is remedied once by the introduction of the $\pi \pi-3 \mathrm{NF}$ (the dashed curve), the introduction of the $V^{\text {phe }}$ again worsens the fit to the data.

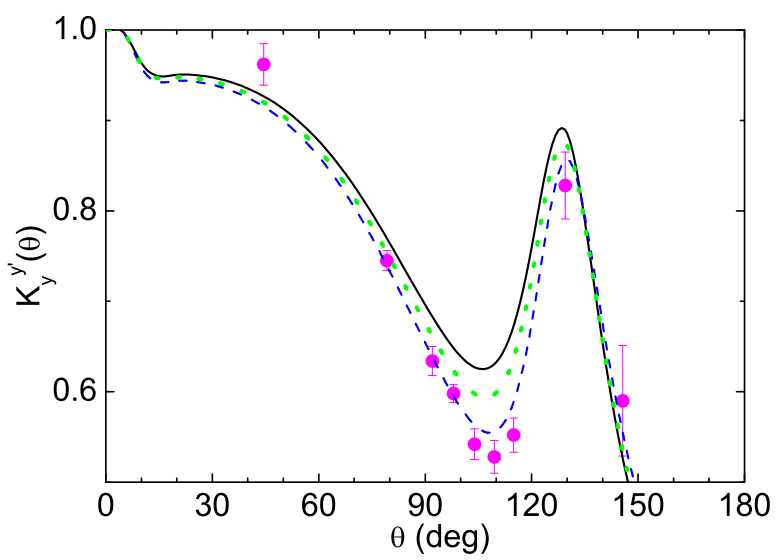

Fig. 2. Nucleon-to-nucleon polarization transfer coefficient $K_{y}^{y^{\prime}}(\theta)$ of the $p d$ elastic scattering at $E_{p}=19.0 \mathrm{MeV}$. The solid (black) curve denotes the calculation with AV18, the dashed (blue) curve that with $\mathrm{AV} 18+\mathrm{BR}_{660}$, and the dotted (green) curve that with $\mathrm{AV} 18+\mathrm{BR}_{800}+V^{\text {phe }}$. Experimental data are taken from Ref. [13].

\section{Pion-sigma exchange three-nucleon force}

In the preceding section, we show that there is a possible $3 \mathrm{NF}$ that remedies the deficiency of the $\pi \pi-3 \mathrm{NF}$. Of course, it is nothing but a phenomenological one and we need to seek a physical $3 \mathrm{NF}$ that produces a tensor component, which might be different from the $\pi \pi-3 \mathrm{NF}$ and play a similar role as the $V^{\text {phe }}$. As a possible mechanism to produce such a $3 \mathrm{NF}$, we consider a diagram shown in Fig. 3, namely so called $\pi-\sigma$ Born diagram [14] $(\pi \sigma-3 \mathrm{NF})$. In the diagram, the vertical line at the intermediate state of the middle nucleon (2) indicates the nucleon Born terms, from which an on-mass-shell nucleon propagation is subtracted to avoid a double counting of the iteration of the nucleonnucleon potential. This diagram is picked up because of a conjecture that the coupling of a pion and a sigma boson might cause a tensor interaction which has a different character caused from the coupling of two pions.

An explicit form of the potential for the diagram of Fig. 3 and its permutation with respect to $(1 \leftrightarrow 2)$ is given as

$$
V\left(\mathbf{r}_{13}, \mathbf{r}_{23}\right)=V_{0}^{\pi \sigma}\left(\tau_{1} \cdot \tau_{2}\right)
$$

$$
\begin{aligned}
& \times\left[\left(\sigma_{1} \cdot \hat{\mathbf{x}}_{12}\right)\left(\sigma_{2} \cdot \hat{\mathbf{x}}_{32}\right) Y_{\pi}^{\prime}\left(x_{12}\right) Y_{\sigma}^{\prime}\left(x_{32}\right)\right. \\
& \left.+\left(\sigma_{2} \cdot \hat{\mathbf{x}}_{21}\right)\left(\sigma_{1} \cdot \hat{\mathbf{x}}_{31}\right) Y_{\pi}^{\prime}\left(x_{21}\right) Y_{\sigma}^{\prime}\left(x_{31}\right)\right],
\end{aligned}
$$

where

$$
\begin{gathered}
V_{0}^{\pi \sigma}=-\frac{g_{\pi}^{2}}{4 \pi} \frac{g_{\sigma}^{2}}{4 \pi} \frac{m_{\pi}^{4}}{4 m_{N}^{3}} \\
\mathbf{x}_{i j}=m_{\pi} \mathbf{r}_{i j}, \\
Y_{b}(x)=\frac{1}{2 \pi^{2}} \int d \mathbf{q} \exp (-\imath \mathbf{q} \cdot \mathbf{x}) \frac{F_{b}\left(q^{2}\right)}{q^{2}+\left(m_{b} / m_{\pi}\right)^{2}},
\end{gathered}
$$

and ' means the differential with respect to $x_{i j}$.

We use the form factor of

$$
F_{b}\left(q^{2}\right)=\left\{\frac{\left(\Lambda_{b} / m_{\pi}\right)^{2}-\left(m_{b} / m_{\pi}\right)^{2}}{q^{2}+\left(\Lambda_{b} / m_{\pi}\right)^{2}}\right\}^{2},
$$

and the mass of $\sigma$ being $m_{\sigma}=500 \mathrm{MeV}$.

When choosing $\Lambda_{\pi}=800 \mathrm{MeV}$ and $\Lambda_{\sigma}=1300 \mathrm{MeV}$, we find that the $\pi \sigma-3 \mathrm{NF}$ with $V_{0}^{\pi \sigma}=-14.9 \mathrm{MeV}$ in conjunction with the AV18-2NF and the Brazil $\pi \pi-3 \mathrm{NF}$ (AV18 $+\mathrm{BR}_{800}+V^{\pi \sigma}$ ) gives the ${ }^{3} \mathrm{He}$ binding energy close to the empirical value. This value of $V_{0}^{\pi \sigma}$ together with $g_{\pi}^{2} / 4 \pi=$ 14.4 gives $g_{\sigma}^{2} / 4 \pi=9.0$, which is consistent with the values used in the Bonn 2NF models.

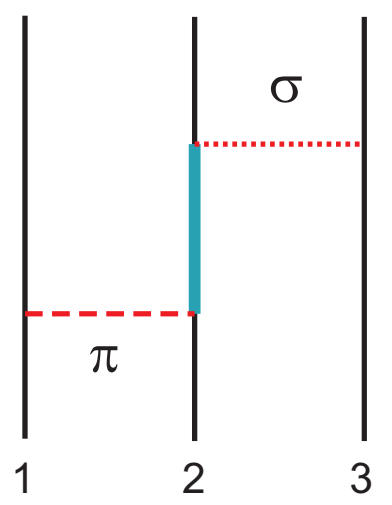

Fig. 3. Born diagram of the pion-sigma exchange three-nucleon force. The vertical solid lines denote nucleons.

In Fig. 4, experimental data [15-18] and calculations for the differential cross sections $\sigma(\theta)$ of the $p d$ elastic scattering at $E_{p}=10.0 \mathrm{MeV}$ are plotted as a ratio to the corresponding calculation with the AV18 potential. First, we have to notice that there are some discrepancies among the available experimental data. Also, the calculation with $\mathrm{AV} 18+\mathrm{BR}_{800}+V^{\pi \sigma}$ reveals a rather large deviation from the AV18 $+\mathrm{RB}_{660}$ calculation. (It is noted that the calculation with $\mathrm{AV} 18+\mathrm{BR}_{800}+V^{\text {phe }}$ almost coincides with the AV18 $+\mathrm{RB}_{660}$ calculation.) At forward angles, while the $\mathrm{AV} 18+\mathrm{RB}_{660}$ calculation is consistent with the Kyushu data [18], the $\mathrm{AV} 18+\mathrm{BR}_{800}+V^{\pi \sigma}$ calculation is rather consistent with the Köln data [17]. On the other hand, at back ward angles, both calculations seem to overestimate the data by $5-10 \%$. Apparently, more precise measurements of the differential cross sections will provide an answer to the question whether such a large effect at forward angles by the $\pi \sigma-3 \mathrm{NF}$ is acceptable or not. 
In Fig. 5, results of the tensor analyzing power $T_{21}(\theta)$ are shown. The effect of the $\pi \sigma-3 \mathrm{NF}$, similarly as the $V^{\text {phe }}$, brings the calculation of $T_{21}(\theta)$ at $\theta=90^{\circ}$ close to the data, which is once shifted away from the experimental data by the effect of the $\pi \pi-3 \mathrm{NF}$.

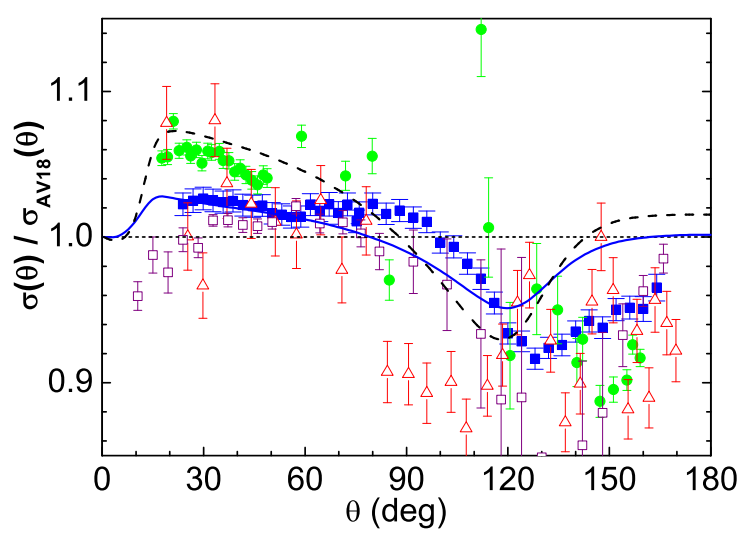

Fig. 4. Ratio of differential cross section of the $p d$ elastic scattering at $E_{p}=10.0 \mathrm{MeV}$ to calculation with the AV18 potential. The solid curve denotes the calculation with $\mathrm{AV} 18+\mathrm{BR}_{660}$, and the dashed curve that with $\mathrm{AV} 18+\mathrm{BR}_{800}+V^{\pi \sigma}$. Experimental data are taken from Ref. [15] (open squares), Ref. [16] (triangles), Ref. [17] (circles), and Ref. [18] (solid squares).

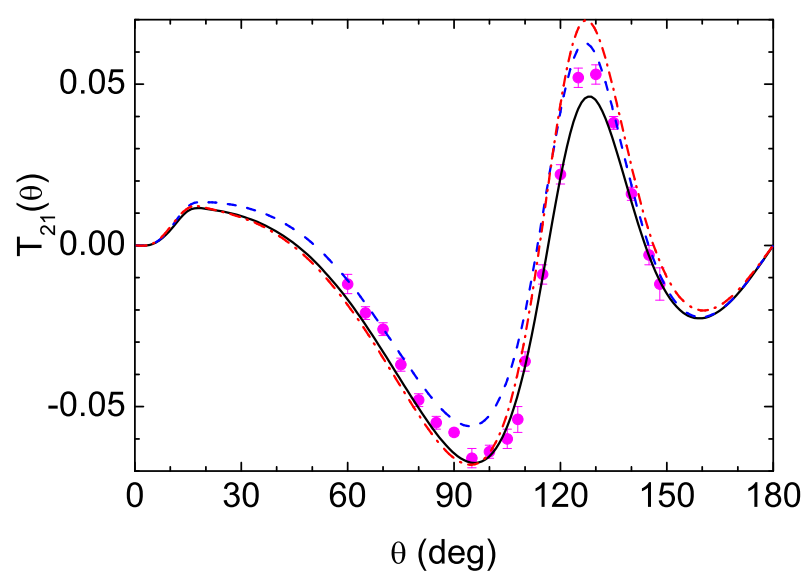

Fig. 5. Tensor analyzing power $T_{21}(\theta)$ of the $p d$ elastic scattering at $E_{p}=10.0 \mathrm{MeV}$ (or equivalently $E_{d}=20.0 \mathrm{MeV}$ ). The solid (black) curve denotes the calculation with AV18, the dashed (blue) curve that with AV18 $+\mathrm{BR}_{680}$, and the dashed-dotted (red) curve one with $\mathrm{AV} 18+\mathrm{BR}_{800}+V^{\pi \sigma}$. Experimental data are taken from Ref. [10].

Finally, results of the nucleon-to-nucleon polarization transfer coefficient $K_{y}^{y^{\prime}}(\theta)$ in ${ }^{2} \mathrm{H}(\vec{p}, \vec{p})^{2} \mathrm{H}$ reaction are plotted in Fig. 6. In contrast to the effect of the $V^{\text {phe }}$, the introduction of the $\pi \sigma-3 \mathrm{NF}$ gives a minor effect and the agreement with the experimental data is still good as the AV18 calculation. This shows that the $\pi \sigma-3 \mathrm{NF}$ gives a similar tensor effect as the $V^{\text {phe }}$ for $T_{21}(\theta)$, but gives different tensor effect for $K_{y}^{y^{\prime}}(\theta)$, which is consistent with the present experimental data.

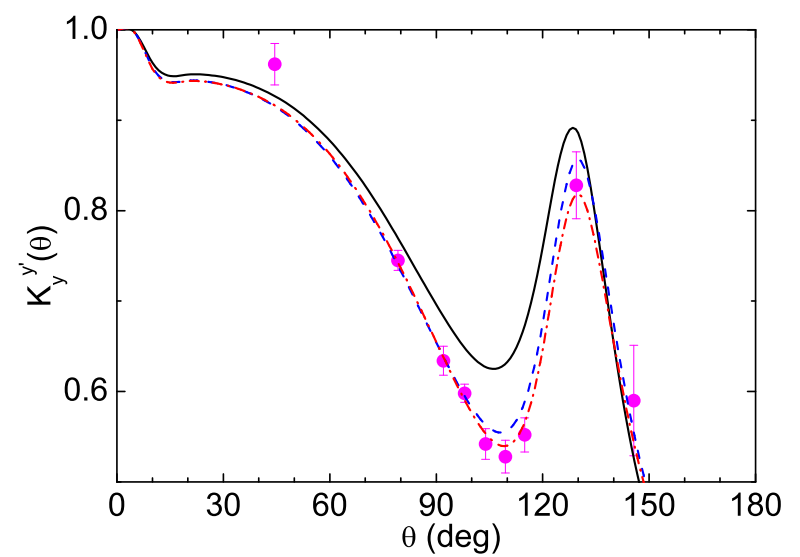

Fig. 6. Nucleon-to-nucleon polarization transfer coefficient $K_{y}^{y^{\prime}}(\theta)$ of the $p d$ elastic scattering at $E_{p}=19.0 \mathrm{MeV}$. The solid (black) curve denotes the calculation with AV18, the dashed (blue) curve that with $\mathrm{AV} 18+\mathrm{BR}_{680}$, and the dashed-dotted (red) curve one with $\mathrm{AV} 18+\mathrm{BR}_{800}+V^{\pi \sigma}$. Experimental data are taken from Ref. [13].

\section{Summary}

We have studied effects of two different 3NF models, phenomenological model and meson-theoretical model, to improve the fit to experimental data of the $p d$ scattering observables that are not successfully explained by the introduction of the $\pi \pi-3 \mathrm{NF}$. The phenomenological $3 \mathrm{NF}$ is composed of (repulsive) central, tensor, and spin-orbit components. As a physical origin of the tensor component in the phenomenological $3 \mathrm{NF}$, the $\pi \sigma-3 \mathrm{NF}$, which is originated from the pion-sigma exchange mechanism, is studied. The $\pi \sigma-3 \mathrm{NF}$ may cancel a wrong behavior of the tensor components in the $\pi \pi-3 \mathrm{NF}$, and as a result, works to explain the tensor analyzing power $T_{21}(\theta)$. The introduction of the $\pi \sigma-3 \mathrm{NF}$ also successfully explains the nucleon-to-nucleon polarization transfer coefficient $K_{y}^{y^{\prime}}(\theta)$. On the other hand, the $\pi \sigma-3 \mathrm{NF}$ gives a large effect in the $N d$ differential cross section at forward angles, which requires more precise data around $10 \mathrm{MeV}$.

The numerical calculations in the present paper are supported by the Research Center for Computing and Multimedia Studies, Hosei University.

\section{References}

1. A. Kievsky, M. Viviani, and S. Rosati, Phys. Rev. C64 (2001) 024002 
2. A. Deltuva, A. C. Fonseca, and P. U. Sauer, Phys. Rev. C71 (2005) 054005

3. T. Sasakawa and T. Sawada, Phys. Rev. C20 (1979) 1954

4. Y. Wu, S. Ishikawa, and T. Sasakawa, Few-Body Syst. 15 (1993) 145

5. S. Ishikawa, Few-Body Syst. 32 (2003) 229

6. S. Ishikawa, Mod. Phys. Lett. A24 (2009) 855

7. S. Ishikawa, Phys. Rev. C80 (2009) 054002

8. R. B. Wiringa, V. G. J. Stokes, and R. Schiavilla, Phys. Rev. C51 (1995) 38

9. S. Ishikawa and M. R. Robilotta, Phys. Rev. C76 (2007) 014006

10. M. Sawada, S. Seki, K. Furuno, Y. Tagishi, Y. Nagashima, J. Schimizu, M. Ishikawa, T. Sugiyama, L. S. Chuang, W. Grüebler, J. Sanada, Y. Koike, and Y. Taniguchi, Phys. Rev. C27 (1983) 1932

11. S. Ishikawa, M. Tanifuji, and Y. Iseri, Phys. Rev. C67 (2003) 061001(R)

12. S. Ishikawa, Phys. Rev. C75 (2007) 061002(R)

13. L. Sydow, S. Lemaitre, P. Niessen, K. R. Nyga, G. Rauprich, R. Reckenfelderbäumer, S. Vohl, H. Paetz gen. Schieck, H. Witała, and W. Glöckle, Nucl. Phys. A567 (1994) 55

14. J. Adam, Jr., M. T. Peña, and A. Stadler, Phys. Rev. C69 (2004) 034008

15. D. C. Kocher and T. B. Clegg, Nucl. Phys. A132 (1969) 455

16. W. Grüebler, V. König, P. A. Schmelzbach, B. Jenny, H. R. Bürgi, P. Doleschall, G. Heidenreich, H. Roser, F. Seiler, and W. Reichart, Phys. Lett. B74 (1978) 173

17. G. Rauprich, H. J. Hähn, M. Karus, P. Nießen, K. R. Nyga, H. Oswald, L. Sydow, H. Paetz gen. Schieck, and Y. Koike, Few-Body Syst. 5 (1988) 67

18. K. Sagara, H. Oguri, S. Shimizu, K. Maeda, H. Nakamura, T. Nakashima, and S. Morinobu, Phys. Rev. C50 (1994) 576 